\title{
Preimplantation Genetic Testing for Thalassemia Through Next- Generation Sequencing of Affected-embryos
}

\author{
Zhanhui Ou \\ Guangzhou Women and Children's Medical Center, Guangzhou Medical University \\ Yu Deng \\ Guangzhou Women and Children's Medical Center, Guangzhou Medical University \\ Yunhao Liang \\ Guangzhou Women and Children's Medical Center, Guangzhou Medical University \\ Zhiheng Chen \\ Guangzhou Women and Children's Medical Center, Guangzhou Medical University \\ Ling Sun ( $\sim$ sunling6299@163.com ) \\ Guangzhou Women and Children's Medical Center, Guangzhou Medical University
}

\section{Research Article}

Keywords: Preimplantation genetic testing, a-thalassemia, $\beta$-thalassemia, SNP linkage, Next- generation sequencing, Affected embryo, Monogenic diseases

Posted Date: December 29th, 2021

DOI: https://doi.org/10.21203/rs.3.rs-1202545/v1

License: (c) (i) This work is licensed under a Creative Commons Attribution 4.0 International License. Read Full License 


\section{Abstract}

Background: To evaluate the ability of next-generation sequencing (NGS) to conduct preimplantation genetic testing (PGT) for thalassemia using affected embryos.

Methods: This study included data from 36 couples who underwent PGT for thalassemia without proband and relative pedigrees. NGS results were compared with prenatal diagnosis results.

Results: Thirty-six couples (29 a-thalassemia and $7 \beta$-thalassemia) underwent 41 PGT cycles (31 a-thalassemia and $10 \beta$-thalassemia). All biopsied blastocysts received conclusive results from NGS analysis $(100 \%, 217 / 217)$. One hundred and sixty $(73.7 \%, 160 / 217)$ were determined to be unaffected by thalassemia. PGT-A (PGT for aneuploidy) results showed that $112(70.0 \%, 112 / 160)$ were euploid. Thirty-four couples were transferred with a single blastocyst (53 frozen embryo transfer (FET) cycles). Thirty-two cycles resulted in clinical pregnancies, and the clinical pregnancy rate was 60.1\% (32/53) per FET cycle. Twenty-two cycles (22 couples) resulted in 23 live births and the live birth rate was $43.4 \%$ ( $23 / 53,3$ cycles were ongoing pregnancy). All 25 cycles' prenatal diagnosis results and/or thalassemia gene analysis after the delivery were concordant with the NGS-PGT results. Seven cycles were miscarried before 12 weeks' gestation, and the abortion villus in four cycles showed a normal karyotype and thalassemia results consistent with the NGSPGT results. Aborted fetus samples from 3 cycles were not available because the pregnancy was less than 5 weeks.

Conclusion: NGS can be used to conduct PGT for thalassemia using affected embryos as a reference.

Trial registration: Retrospectively registered.

\section{Introduction}

Thalassemia, one of the most common monogenic diseases, is an inherited blood disorder characterized by a reduction in the synthesis of hemoglobin (HB) subunit $\mathrm{a}$ or $\beta$ (HB a or $\beta$ chain). In southern China, the prevalence of $\alpha$-thalassemia and $\beta$-thalassemia are $8.53 \%$ and $2.54 \%$, respectively (1). This genetically inherited disease has threatened the lives of millions of people for decades and no effective treatments are available. Homozygotes with the Southeast Asian (SEA) deletion develop Hb Bart's hydrops fetalis syndrome and they usually die either late in gestation or a few minutes after birth (2). Infants with severe forms of $\beta$-thalassemia can now survive but require much medical intervention, resulting in a rising global economic and healthcare burden (3).

Prenatal diagnosis is advocated in China to prevent the birth of babies with severe thalassemia. However, it requires an invasive procedure that may induce miscarriage, and may result in the mental burden of terminating an affected pregnancy. At present, preimplantation genetic testing for monogenic disease (PGT-M) is a method that can effectively prevent thalassemia in the children of couples who are at risk of transmitting this genetic condition to their offspring $(4,5)$.

Since the early 1990s, PGT-M has been used for X-linked genetic diseases (6), with PCR-based methods being used in past decades. However, allele dropout (ADO) is a main cause of misdiagnosis in PGT-M so a direct PCR approach cannot be used as the sole method for diagnosis and detection of the target pathogenic mutation sites (7). In recent years, linkage analysis has been widely used to increase PGT-M accuracy (8). This method relies on short tandem repeat (STR), or single nucleotide polymorphism (SNP) markers linked to the mutations, but in some cases with de novo mutations or lack of proband, haplotypes cannot be constructed.

This has made some inherited genetic diseases, like a-thalassemia difficult to detect with PGT. This is a serious issue as babies with this condition usually die immediately after birth. Sometimes couples have discovered their status as carriers in the preoperative examination before in vitro fertilization (IVF) treatment. Thus, there is a particularity in thalassemia concerning the haplotypes. In recent years, constructing the haplotypes through both parents has been a common approach, but this method is tedious and expensive. Another method is through single sperm and polar body diagnosis using NGS when lacking relatives or proband, which is also used in clinics $(9,10)$. However, this method requires an extra biopsy to collect polar bodies, and often needs multiple single sperms, which is tedious and expensive. Recently, some studies have explored constructing the haplotypes through affected embryos with NGS $(4,11,12)$. But since these studies only had one or two cases, this method requires further validation.

In this study, we conducted PGT for thalassemia using SNP haplotyping with affected embryos as a reference. To the best of our knowledge, this is the largest study to use PGT-M for thalassemia without the relatives and proband in a clinic.

\section{Materials And Methods}

\section{Patients}

This study was approved by the Reproductive Medical Ethics Committee of Guangzhou Women and Children's Hospital. Thirty-six couples where both parents carried genes for either a-thalassemia (29 couples) or $\beta$-thalassemia (7 couples) were selected at the Guangzhou Women and Children's Hospital between June 2017 and June 2021. Written informed consent was obtained from each couple.

\section{Blastocyst biopsy and vitrification}


We performed standard ovarian stimulation, intracytoplasmic sperm injection (ICSI), embryo culture, and blastocyst vitrification for each of the 36 couples as previously reported $(12,13)$. Biopsy was performed on day five or six depending on the blastocyst grade on the day of biopsy $(7)$.

\section{Whole- genome amplification (WGA)}

Multiple displacement amplification (MDA) using a REPLI-g Single Cell Kit (Qiagen, Germany) was performed according to the manufacturer's protocol. The WGA products were purified and then sequenced. All PCR amplifications were performed on a 96 Well Thermal Cycler Veriti DX (Life Technologies). All procedures were carried out in accordance with the manufacturer's protocol and, as previously reported (12).

\section{NGS sequencing and haplotype construction}

Mutation and SNP sites were submitted to lon Ampliseq Designer (https://www.ampliseq.com/) for primer design. Overall, 138 SNPs within 1 Mb upstream and 132 SNPs within 2 Mb downstream of the mutation gene (chr16:215400-234700 NM_000517.4 (HBA2) and NM_000558.4 (HBA1)) were selected for NGS-based a-thalassemia SNP haplotyping. Eighty-five SNP markers located either $1 \mathrm{Mb}$ upstream or downstream of the mutation gene (chr11:52466965248301 NM_000518.4 (HBB)) were selected for NGS-based $\beta$-thalassemia SNP haplotyping. Only the SNPs that were heterozygous in one parent and homozygous in their parent were considered as informative SNPs. The genomic DNA of the couple and the WGA products were amplified with these primers for haplotype construction. Sequencing libraries were prepared using the sequencing library kit (NEXTflex Rapid DNA-seq Kit 96rxns, BIOO), and the libraries were sequenced on an Illumina MiseqDX platform (Illumina) using a MiSeq Dx Reagent Kit V3 (IIlumina). All procedures were carried out in accordance with the manufacturer's protocol. The sequencing data were analyzed by Peking Jabrehoo Med Tech., Ltd. Copy number variations (CNV) analysis for aneuploidy testing was performed as previously described (13).

\section{Frozen embryo transfer and follow up}

Hormone replacement therapy was used to prepare the uterus endometrium. A frozen non-pathogenic blastocyst with euploid karyotype was thawed and cultured for 2 hours before being transferred into the uterus as previously described (14). Clinical pregnancies (CP) were defined after observation of a gestational sac with or without a fetal heartbeat on ultrasound evaluation 4 weeks after frozen embryo transfer (FET). Clinical miscarriage was determined to occur when a pregnancy failed to progress after an intrauterine gestational sac had been detected with pelvic ultrasonography. Amniocentesis was performed at about 17 weeks of gestation and/or gene detection of the blood was performed after birth to verify the consistency with the PGT.

\section{Statistical analysis}

Statistical analysis was performed using SPSS software v. 19 for Windows (SPSS Inc., Chicago, USA), applying parametric and nonparametric tests when appropriate. Continuous variables were expressed as mean \pm standard deviation (SD) and analyzed using Student's t-test. Categorical variables were expressed as percentages and analyzed using $\chi^{2}$ or Fisher's exact test depending on the sample size. Statistical significance was defined as p-values less than 0.05 .

\section{Results}

\section{Trophectoderm biopsy and WGA}

The 36 couples ( 29 a-thalassemia and $7 \beta$-thalassemia) underwent a total of 41 PGT cycles ( 31 a-thalassemia and $10 \beta$-thalassemia) (Table 1 and Supplementary Table 1) (Figure 1). Seven hundred and seventy-seven oocytes were retrieved. Of these 631 were fertilized with intracytoplasmic sperm injection (ICSI), and 217 were cultured to blastocysts of good enough quality to perform trophectoderm (TE) biopsy (better than $₫ \mathrm{CC}$ ). The average number of blastocysts for each couple was 6.0 (217/36). WGA was successfully performed for all TE cells.

\section{NGS-based SNP haplotyping and mutation detection}

Due to these families lacking related relatives and proband, all analyses were based on the blastocysts. Three couples (families 8,31 , and 35 ) only had two biopsied blastocysts and did not receive conclusive PGT results from NGS. A second PGT cycle produced conclusive results. Therefore, all of the biopsied blastocysts received conclusive PGT results $(100 \%, 217 / 217)$ (Table 1).

Using family 13 as an example to analyze a- thalassemia (Figure 2), 138 SNPs within $1 \mathrm{Mb}$ upstream and 132 SNPs within 2 Mb downstream from the HBA1 and HBA2 gene were adopted with sequencing depth $>30 \mathrm{X}$. We could deduce whether the mutation allele was present in the embryo by analyzing these SNPs. For instance, we found that embryos 5 and 7 carried the disease allele from the couple according to the sequencing depth of the SEA area (Figure 2a, part of the SNP results). We also found that these two embryos inherited both maternal and paternal disease-associated haplotypes. Next, information SNPs in the SEA area were used to construct the haplotype. In brief, the mother was heterozygous A/C, and the father was A/A on SNP in position 119006. This SNP was considered as the maternal information SNP. While the affected embryos (5 and 7) were homozygous A/A (Figure $2 b$, part of the SNP results), we could easily deduce that alleles with the base A from the mother were pathogenic, and this was the disease-associated haplotype. 
At least two upstream and two downstream markers closely linked to the gene underlying the mutation were analyzed, and the disease-associated and nondisease-associated maternal haplotype was successfully distinguished. Hence, we concluded that embryos 1, 5, and 7 carried the disease-associated maternal haplotype. Similarly, in position 207611, the maternal was C/C and the paternal was C/T, this SNPs was considered as the paternal information SNPs (Figure $2 \mathrm{~b}$ ). While the affected embryos ( 5 and 7 ) were homozygous $\mathrm{C} / \mathrm{C}$. We could easily deduce that alleles with base $\mathrm{C}$ from the father were pathogenic, and this was another disease-associated haplotype. Hence, we concluded that embryos 2, 3, 4, 5, and 7 carried the disease-associated paternal haplotype. So, embryos 5 and 7 were homozygous, embryos 1, 2, 3, and 4 were heterozygous, and embryos 6, 8, and 9 were wildtype.

The analysis method for $\beta$ - thalassemia (using family 30 as an example) is shown in Figure 3. Ninety-five SNPs within 2 Mb upstream and downstream respective from the $H B B$ gene were adopted with sequencing depth $>30 \mathrm{X}$. At first, we could deduce embryos 4 and 6 carried the disease allele according to the sequencing depth of the $\beta C D 41-42$ (Figure 3a). Thus, we deduced that these two embryos inherited both maternal and paternal disease-associated haplotypes (Figure $3 b$ ). Next, information SNPs in the $H B B$ were used to construct the haplotype as described above. We could also conclude that embryos $1,4,5$, and 6 carried the disease-associated maternal haplotype and that embryos 2, 4, and 6 carried the disease-associated paternal haplotype. So, embryos 4 and 6 were homozygous, embryos 1, 2, and 5 were heterozygous, and embryo 3 was wild-type.

\section{PGT results}

After NGS-based SNP haplotyping and mutation detection, $160(73.7 \%, 160 / 217)$ blastocysts were found to be unaffected by either a- thalassemia or $\beta$ thalassemia (Table 1). PGT-A results of these blastocysts showed that $112(70.0 \%, 112 / 160)$ were euploid, which were defined as transferable blastocysts (Table 1). And the average number of transferable blastocysts for each couple was $3.1(112 / 36)$.

Table 1

The preimplantation genetic testing outcomes of the 36 families. 


\begin{tabular}{|c|c|c|c|c|c|c|c|c|c|c|c|c|}
\hline & \multicolumn{2}{|c|}{ Cycles } & \multicolumn{3}{|c|}{ Embryo state } & \multicolumn{2}{|l|}{ PGT results } & \multicolumn{2}{|c|}{$\begin{array}{l}\text { Pregnancy } \\
\text { results }\end{array}$} & \multicolumn{2}{|l|}{ Genetic testing } & \multirow[b]{2}{*}{ After born } \\
\hline & PGT & FET & Oocyte & $\mathrm{M} \otimes$ & $\begin{array}{l}\text { Biopsy } \\
\text { blastocyst }\end{array}$ & Unaffected $^{1}$ & Transferrable ${ }^{2}$ & $\mathrm{CP}$ & LB & PGT-M & Amniocentesis & \\
\hline $\begin{array}{l}\text { Family } \\
1\end{array}$ & 1 & 1 & 26 & 25 & 9 & 6 & 3 & 1 & 1 & Wild type & Wild type & Wild type \\
\hline $\begin{array}{l}\text { Family } \\
2\end{array}$ & 1 & 1 & 22 & 17 & 8 & 6 & 4 & 1 & 1 & Heterozygote & Heterozygote & Heterozygote \\
\hline $\begin{array}{l}\text { Family } \\
3\end{array}$ & 1 & 1 & 13 & 12 & 3 & 2 & 1 & 1 & 1 & Wild type & NA & Wild type \\
\hline $\begin{array}{l}\text { Family } \\
4\end{array}$ & 1 & 1 & 11 & 10 & 5 & 2 & 2 & 1 & 1 & Wild type & NA & Wild type \\
\hline $\begin{array}{l}\text { Family } \\
5\end{array}$ & 1 & 1 & 12 & 12 & 9 & 6 & 3 & 1 & 1 & Heterozygote & Heterozygote & Heterozygote \\
\hline $\begin{array}{l}\text { Family } \\
6\end{array}$ & 1 & 2 & 15 & 14 & 7 & 6 & 2 & 1 & 1 & Heterozygote & Heterozygote & Heterozygote \\
\hline $\begin{array}{l}\text { Family } \\
7\end{array}$ & 1 & 1 & 24 & 20 & 4 & 4 & 1 & 1 & 1 & Heterozygote & Heterozygote & Heterozygote \\
\hline $\begin{array}{l}\text { Family } \\
8^{3}\end{array}$ & 2 & 2 & 44 & 31 & 8 & 6 & 4 & 1 & 1 & Heterozygote & NA & Heterozygote \\
\hline $\begin{array}{l}\text { Family } \\
9\end{array}$ & 1 & 2 & 35 & 27 & 6 & 6 & 6 & 1 & 0 & Wild type & Miscarriage & NA \\
\hline $\begin{array}{l}\text { Family } \\
10\end{array}$ & 1 & 1 & 28 & 16 & 4 & 1 & 1 & 1 & 0 & Wild type & Miscarriage & NA \\
\hline $\begin{array}{l}\text { Family } \\
11\end{array}$ & 1 & 1 & 15 & 11 & 5 & 3 & 2 & 1 & 1 & Wild type & Wild type & NA \\
\hline $\begin{array}{l}\text { Family } \\
12\end{array}$ & 1 & 3 & 21 & 16 & 4 & 4 & 3 & 1 & 1 & Heterozygote & NA & Heterozygote \\
\hline $\begin{array}{l}\text { Family } \\
13\end{array}$ & 1 & 2 & 26 & 20 & 9 & 7 & 5 & 1 & 1 & Wild type & Wild type & NA \\
\hline $\begin{array}{l}\text { Family } \\
14\end{array}$ & 1 & 2 & 7 & 5 & 3 & 1 & 1 & 0 & 0 & Heterozygote & NA & NA \\
\hline $\begin{array}{l}\text { Family } \\
15^{4}\end{array}$ & 2 & 1 & 48 & 36 & 11 & 7 & 2 & 1 & 2 & Wild type & NA & Wild type \\
\hline $\begin{array}{l}\text { Family } \\
16\end{array}$ & 1 & 1 & 19 & 12 & 4 & 3 & 2 & 1 & 1 & Heterozygote & Heterozygote & NA \\
\hline $\begin{array}{l}\text { Family } \\
17\end{array}$ & 1 & 0 & 6 & 4 & 2 & 0 & 0 & 0 & 0 & NA & NA & NA \\
\hline $\begin{array}{l}\text { Family } \\
18\end{array}$ & 1 & 1 & 19 & 10 & 3 & 2 & 2 & 1 & 1 & Heterozygote & Heterozygote & NA \\
\hline $\begin{array}{l}\text { Family } \\
19\end{array}$ & 1 & 3 & 40 & 33 & 15 & 11 & 11 & 1 & 1 & Heterozygote & NA & Heterozygote \\
\hline $\begin{array}{l}\text { Family } \\
20\end{array}$ & 1 & 2 & 25 & 21 & 4 & 4 & 3 & 1 & 1 & Heterozygote & NA & Heterozygote \\
\hline $\begin{array}{l}\text { Family } \\
21\end{array}$ & 1 & 1 & 19 & 14 & 8 & 8 & 4 & 1 & 1 & Wild type & Wild type & NA \\
\hline $\begin{array}{l}\text { Family } \\
22\end{array}$ & 1 & 1 & 19 & 13 & 5 & 5 & 3 & 1 & 1 & Heterozygote & Heterozygote & NA \\
\hline $\begin{array}{l}\text { Family } \\
23\end{array}$ & 1 & 1 & 29 & 27 & 6 & 4 & 4 & 1 & 0 & Heterozygote & NA & NA \\
\hline $\begin{array}{l}\text { Family } \\
24\end{array}$ & 1 & 1 & 16 & 15 & 7 & 4 & 4 & 1 & 1 & Heterozygote & Heterozygote & NA \\
\hline $\begin{array}{l}\text { Family } \\
25\end{array}$ & 1 & 1 & 19 & 19 & 5 & 3 & 2 & 1 & 0 & Wild type & Miscarriage & NA \\
\hline $\begin{array}{l}\text { Family } \\
26\end{array}$ & 1 & 2 & 8 & 7 & 4 & 4 & 4 & 1 & 0 & Wild type & Miscarriage & NA \\
\hline $\begin{array}{l}\text { Family } \\
27\end{array}$ & 1 & 2 & 11 & 9 & 6 & 5 & 3 & 1 & 0 & Wild type & Miscarriage & NA \\
\hline
\end{tabular}

Page 5/11 


\begin{tabular}{|c|c|c|c|c|c|c|c|c|c|c|c|c|}
\hline $\begin{array}{l}\text { Family } \\
28\end{array}$ & 1 & 1 & 11 & 11 & 3 & 2 & 1 & 0 & 0 & Wild type & NA & NA \\
\hline $\begin{array}{l}\text { Family } \\
29\end{array}$ & 1 & 2 & 17 & 13 & 5 & 3 & 3 & 0 & 0 & Wild type & NA & NA \\
\hline $\begin{array}{l}\text { Family } \\
30\end{array}$ & 2 & 4 & 47 & 40 & 10 & 7 & 6 & 2 & 0 & Wild type & Miscarriage & NA \\
\hline $\begin{array}{l}\text { Family } \\
31^{3}\end{array}$ & 2 & 3 & 27 & 26 & 7 & 6 & 5 & 1 & 1 & Heterozygote & Heterozygote & Heterozygote \\
\hline $\begin{array}{l}\text { Family } \\
32\end{array}$ & 1 & 2 & 21 & 15 & 6 & 5 & 5 & 1 & 1 & Heterozygote & Heterozygote & Heterozygote \\
\hline $\begin{array}{l}\text { Family } \\
33\end{array}$ & 1 & 0 & 15 & 14 & 3 & 1 & 0 & 0 & 0 & NA & NA & NA \\
\hline $\begin{array}{l}\text { Family } \\
34\end{array}$ & 1 & 1 & 12 & 11 & 9 & 7 & 7 & 1 & 1 & Wild type & NA & Wild type \\
\hline $\begin{array}{l}\text { Family } \\
35^{3}\end{array}$ & 2 & 1 & 28 & 28 & 4 & 4 & 1 & 1 & 0 & Heterozygote & Heterozygote & NA \\
\hline $\begin{array}{l}\text { Family } \\
36\end{array}$ & 1 & 1 & 22 & 17 & 6 & 5 & 2 & 1 & 0 & Heterozygote & Heterozygote & NA \\
\hline \multicolumn{13}{|c|}{$\begin{array}{l}\text { PGT: preimplantation genetic testing, FET: Frozen embryo transfer, CP: clinical pregnancy, LB: live birth, NA: not applicable } \\
1 \text { Unaffected embryos, including non-carrier and carrier embryos }\end{array}$} \\
\hline
\end{tabular}

\section{Clinical outcomes}

Two couples had no transferable blastocysts after the first PGT cycle, and did not perform another PGT cycle. The other 34 couples were transferred with a single blastocyst (53 FET cycles). Thirty-two cycles resulted in clinical pregnancy and the clinical pregnancy rate was $60.1 \%(32 / 53)$ per FET cycle. Family 15 developed a monochorionic diamniotic twin pregnancy after a single blastocyst transfer and resulted in the birth of two healthy babies.

Twenty-two cycles (22 couples) resulted in 23 live births and the live birth rate was $43.4 \%$ (23/53, 3 cycles were ongoing pregnancy). The prenatal diagnosis results and/or thalassemia gene analysis after the delivery were concordant with the NGS-PGT results for all 25 cycles. Seven cycles resulted in miscarriage before 12 weeks' gestation (7/32, 21.9\%), and the abortion villus from 4 of the cycles showed normal karyotype and thalassemia results consistent with the NGS-PGT results. However, samples from the aborted fetuses' in 3 cycles were not available because the pregnancy lasted less than 5 weeks.

\section{Discussion}

The small amount of DNA taken from biopsied trophectoderm cells and amplification bias based on WGA can lead to ADO (15). And PCR-based methods for PGT are inevitably affected by ADOs. Although haplotype analysis with short tandem repeat (STR) may reduce the effects of ADO, the number of STR loci is limited. Further, recombination between STR loci and target genes may affect the diagnosis accuracy $(16,17)$. Hence, single-nucleotide polymorphisms (SNPs) linked to the mutated genes are being used more and more to establish haplotype linkages in clinical practice (18). However, this technique requires proband or the pedigrees of the parents to construct the haplotype for linkage analysis. This makes performing PGT difficult for detecting some inherited genetic diseases like a-thalassemia.

At present, the most widely used method for PGT-M without pedigrees is single-sperm for haplotyping, namely, isolating a single sperm and analyzing the genotypes of SNP alleles via NGS $(10,19)$. However, polymorphic markers need to be identified before linkage analysis and this requires multiple steps and extra laboratory work. This method is usually suitable for dominant inherited genetic diseases from the paternal parent, such as Osteogenesis imperfecta (10). However, both of a-thalassemia and $\beta$-thalassemia are recessive inherited genetic diseases. Thus, using single-sperm for haplotyping would be tedious and cannot identify the maternal haplotype unless polar bodies are also biopsied.

NGS-based PGT can simultaneously detect target mutation sites and linked SNPs, making it possible to provide multiple diagnostic results with the advantages of high accuracy, and throughput (20). Thus, we chose to use affected embryos as a reference to perform haplotype construction in order to avoid the multiple steps. All biopsied blastocysts received conclusive PGT results. In fact, this method is very useful in a clinic as many monogenetic inherited genetic diseases lack intact pedigrees, such as our previous case report PGT-M for Marfan syndrome (12). A previous study by Ren et al. (11) successfully carried out PGT-M based on a mutated allele by sequencing with aneuploidy and linkage analyses for two carrier families with children 
affected with spinal muscular atrophy. They found that this method can correctly diagnose embryos by using affected embryos as the probands. Another study by Chen et al. (4) also found that NGS-based haplotyping can be performed by directly detecting mutation sites and using affected embryos as probands for PGT-M. Li et al. (21) successfully applied the linked-read sequencing method to construct parental haplotypes without recruiting additional family members in two families with alpha thalassemia and the other with NDP gene disorder. However, these studies only used one or two couples. Thus, it is necessary to conduct studies with more samples to validate the use of affected embryos as the probands in a clinic.

In this study, 29 couples with a-thalassemia and 7 couples with $\beta$-thalassemia successfully underwent PGT using affected embryos as a reference. All 217 biopsied blastocysts received conclusive PGT results. After a single blastocyst was transferred in 53 cycles, 32 cycles resulted in clinical pregnancy, and 22 cycles (23 babies) resulted in a live birth. All prenatal diagnosis results and/or thalassemia gene analysis after delivery were concordant with the NGS-PGT results. Therefore, we successfully conducted PGT for thalassemia using SNP haplotyping with affected embryos as a reference. This technique is very useful for some couples with other monogenetic diseases who need to perform PGT without proband and his/her parents' pedigrees.

Although there are challenges associated with ADO, haplotyping linkage analysis with more informative SNPs could help to avoid this. NGS-based PGT can detect the mutated gene directly and construct haplotypes with SNPs close to the mutated gene using PGT to determine ADO and prevent misdiagnosis. Moreover, haplotyping can also be used to distinguish the chromosome of the pathogenic gene from normal chromosomes and find monosomies of chromosomes to avoid misdiagnosis. In our study, more than 100 SNP markers within $1 \mathrm{Mb}$ upstream and downstream of the pathogenic mutation site were used to establish the haplotype. By analyzing these SNPs, we could determine the disease-carrying allele state of each embryo.

The main limitation of this method is that patients may not obtain sufficient embryos as a reference, requiring another oocyte pick-up cycle. Additionally, marriages in proximity may not lead to enough informative SNPs to establish haplotyping.

\section{Conclusions}

NGS can be used to conduct PGT for thalassemia using affected embryos as a reference. Additionally, this method could also be used to perform PGT for other monogenic diseases in the absence of proband and the parents' pedigrees.

\section{Declarations}

\section{Ethics approval and consent to participate}

The study has been approved by the Ethics Committee of our Institution (2021167A01). Written consent was obtained for all willing participants prior to registering for this study. Patient Informed consent to participate in this study.

\section{Consent for publication}

The authors have consented for publication.

\section{Availability of data and materials}

Data were obtained from the referenced publications. Further information is available by contacting Dr. Ou at zhanhui-ou@hotmail.com.

\section{Competing interests}

The authors declare that they have no competing interests.

\section{Funding}

This study was supported by institutional funding of Health Science and technology project of Guangzhou (20201A011029 and 20211A011026).

\section{Authors' contributions}

ZHO developed the concept of the study; All authors contributed to data accumulation; ZHO, YD, YHL and ZHC contributed to data analysis; ZHO wrote the manuscript. All authors contributed to revisions of the manuscript, and approved of the final submission. ZHO takes responsibility for the accuracy of the data analysis.

\section{Acknowledgments}

Thanks to all the peer reviewers for their opinions and suggestions. We also thanks to the company of Peking Jabrehoo Med Tech., Ltd. from China for some technologic helps. 


\section{Author details}

1 Center of Reproductive Medicine, Guangzhou Women and Children's Medical Center, Guangzhou Medical University, 510623, Guangzhou, Guangdong, P.R. China.

\section{References}

1. Xu XM, Zhou YQ, Luo GX, Liao C, Zhou M, Chen PY, et al. The prevalence and spectrum of alpha and beta thalassaemia in Guangdong Province: implications for the future health burden and population screening. Journal of clinical pathology. 2004;57(5):517-22.

2. Taher AT, Weatherall DJ, Cappellini MD. Thalassaemia. Lancet. 2018;391(10116):155-67.

3. Weatherall DJ. Thalassemia as a global health problem: recent progress toward its control in the developing countries. Annals of the New York Academy of Sciences. 2010;1202:17-23.

4. Chen D, Shen X, Wu C, Xu Y, Ding C, Zhang G, et al. Eleven healthy live births: a result of simultaneous preimplantation genetic testing of alpha- and beta-double thalassemia and aneuploidy screening. Journal of assisted reproduction and genetics. 2020;37(3):549-57.

5. Chen D, Shen X, Xu Y, Ding C, Ye Q, Zhong Y, et al. Successful four-factor preimplantation genetic testing: alpha- and beta-thalassemia, human leukocyte antigen typing, and aneuploidy screening. Systems biology in reproductive medicine. 2021;67(2):151-9.

6. Handyside AH, Kontogianni EH, Hardy K, Winston RM. Pregnancies from biopsied human preimplantation embryos sexed by Y-specific DNA amplification. Nature. 1990;344(6268):768-70.

7. Harton GL, De Rycke M, Fiorentino F, Moutou C, SenGupta S, Traeger-Synodinos J, et al. ESHRE PGD consortium best practice guidelines for amplification-based PGD. Human reproduction. 2011;26(1):33-40.

8. Thornhill AR, Handyside AH, Ottolini C, Natesan SA, Taylor J, Sage K, et al. Karyomapping-a comprehensive means of simultaneous monogenic and cytogenetic PGD: comparison with standard approaches in real time for Marfan syndrome. Journal of assisted reproduction and genetics. 2015;32(3):347-56.

9. Wu H, Shen X, Huang L, Zeng Y, Gao Y, Shao L, et al. Genotyping single-sperm cells by universal MARSALA enables the acquisition of linkage information for combined pre-implantation genetic diagnosis and genome screening. Journal of assisted reproduction and genetics. 2018;35(6):10718.

10. Chen L, Diao Z, Xu Z, Zhou J, Yan G, Sun H. The clinical application of single-sperm-based SNP haplotyping for PGD of osteogenesis imperfecta. Systems biology in reproductive medicine. 2019;65(1):75-80.

11. Ren Y, Zhi X, Zhu X, Huang J, Lian Y, Li R, et al. Clinical applications of MARSALA for preimplantation genetic diagnosis of spinal muscular atrophy. Journal of genetics and genomics = Yi chuan xue bao. 2016;43(9):541-7.

12. Deng Y, Ou Z, Li R, Chen Z, Liang P, Sun L. Affected-embryo-based SNP haplotyping with NGS for the preimplantation genetic testing of Marfan syndrome. Systems biology in reproductive medicine. 2021;67(4):298-306.

13. Ou Z, Chen Z, Yin M, Deng Y, Liang Y, Wang W, et al. Re-analysis of whole blastocysts after trophectoderm biopsy indicated chromosome aneuploidy. Human genomics. 2020;14(1):3.

14. Wang H, Ou Z, Chen Z, Yang L, Sun L. Influence of different post-thaw culture time on the clinical outcomes of different quality embryos. Advances in clinical and experimental medicine : official organ Wroclaw Medical University. 2019;28(4):523-7.

15. Harper JC, Wilton L, Traeger-Synodinos J, Goossens V, Moutou C, SenGupta SB, et al. The ESHRE PGD Consortium: 10 years of data collection. Human reproduction update. 2012;18(3):234-47.

16. Natesan SA, Bladon AJ, Coskun S, Qubbaj W, Prates R, Munne S, et al. Genome-wide karyomapping accurately identifies the inheritance of single-gene defects in human preimplantation embryos in vitro. Genetics in medicine : official journal of the American College of Medical Genetics. 2014;16(11):838-45.

17. Gueye NA, Jalas C, Tao X, Taylor D, Scott RT, Jr., Treff NR. Improved sensitivity to detect recombination using qPCR for Dyskeratosis Congenita PGD. Journal of assisted reproduction and genetics. 2014;31(9):1227-30.

18. Chen L, Diao Z, Xu Z, Zhou J, Yan G, Sun H. The clinical application of NGS-based SNP haplotyping for PGD of Hb H disease. Systems biology in reproductive medicine. 2017;63(3):212-7.

19. Altarescu G, Brooks B, Kaplan Y, Eldar-Geva T, Margalioth EJ, Levy-Lahad E, et al. Single-sperm analysis for haplotype construction of de-novo paternal mutations: application to PGD for neurofibromatosis type 1. Human reproduction. 2006;21(8):2047-51.

20. Chamayou S, Sicali M, Lombardo D, Alecci C, Ragolia C, Maglia E, et al. Universal strategy for preimplantation genetic testing for cystic fibrosis based on next-generation sequencing. Journal of assisted reproduction and genetics. 2019.

21. Li Q, Mao Y, Li S, Du H, He W, He J, et al. Haplotyping by linked-read sequencing (HLRS) of the genetic disease carriers for preimplantation genetic testing without a proband or relatives. BMC medical genomics. 2020;13(1):117.

\section{Figures}


Figure 2a

\begin{tabular}{|c|c|c|c|c|c|c|c|c|c|c|c|c|c|}
\hline & start & end & $\mathrm{M}$ & $\mathrm{F}$ & E1 & E2 & E3 & E4 & E5 & E6 & E7 & E8 & E9 \\
\hline IHA_SEA chr16 & 216815 & 216989 & 313 & 397 & 335 & 1098 & 782 & $6 \%$ & 2 & 1673 & 2 & 1278 & 1319 \\
\hline THA_SEA chir16 & 216978 & 217122 & 299 & 556 & 444 & 1156 & 1135 & 1099 & 5 & 2363 & 11 & 1870 & 2136 \\
\hline THA_SEA chr16 & 217111 & 217320 & 113 & 139 & 123 & 329 & 293 & 218 & 0 & 548 & 1 & 392 & 577 \\
\hline THA_SEA chrl 6 & 217306 & 217443 & 2672 & 3691 & 1429 & 3435 & 2801 & 310 & 8 & 6689 & 9 & 6568 & 5771 \\
\hline IHA_SEA carl 16 & 217432 & 217659 & 91 & 151 & 150 & 391 & 407 & 283 & 0 & 623 & 0 & 514 & 660 \\
\hline IHA_SEA chrr16 & 217639 & 217803 & 458 & 726 & 503 & 1286 & 1108 & 1071 & 6 & 1866 & 4 & 1869 & 1978 \\
\hline THA_SEA chrl 6 & 217787 & 217882 & 423 & 432 & 314 & 615 & 488 & 635 & 6 & 1874 & 0 & 1268 & 1277 \\
\hline THA_SEA chrir 6 & 218278 & 218461 & 2400 & 3767 & 2932 & 8284 & 5802 & 6790 & 24 & 12045 & 29 & 12269 & 11966 \\
\hline IHA_SEA chrl 6 & 218449 & 218636 & 56 & 95 & 122 & 310 & 267 & 202 & 1 & 450 & 4 & 382 & 507 \\
\hline THA_SEA chrr 6 & 218625 & 218838 & 79 & 161 & 189 & 461 & 454 & 321 & 1 & 575 & 0 & 566 & 630 \\
\hline
\end{tabular}

\section{Figure 1}

Flow chart and the pregnancy outcomes of the study. 
Figure 2a

\begin{tabular}{|c|c|c|c|c|c|c|c|c|c|c|c|c|c|}
\hline & start & end & M & $\mathrm{F}$ & E1 & E2 & E3 E4 & E5 & E6 & E & E8 & $\mathrm{E}$ & \\
\hline IHA_SEA chr16 & 216815 & 216989 & 313 & 397 & 335 & 1098 & 782 & 696 & 2 & 1673 & 2 & 1278 & 1319 \\
\hline THA_SEA chr16 & 216978 & 217122 & 299 & 556 & 444 & 1156 & 1135 & 1099 & 5 & 2363 & 11 & 1870 & 2136 \\
\hline THA_SEA chr 16 & 217111 & 217320 & 113 & 139 & 123 & 329 & 293 & 218 & 0 & 548 & 1 & 392 & 577 \\
\hline THA_SEA chrl6 6 & 217306 & 217443 & 2672 & 3691 & 1429 & 3435 & 2801 & 3170 & 8 & 6689 & 9 & 6568 & 5771 \\
\hline THA_SEA chr16 & 217432 & 217659 & 91 & 151 & 150 & 391 & 407 & 283 & 0 & 623 & 0 & 514 & 660 \\
\hline THA_SEA chr16 & 217639 & 217803 & 458 & 726 & 503 & 1286 & 1108 & 1071 & 6 & 1866 & 4 & 1869 & 1978 \\
\hline THA_SEA chrl6 6 & 217787 & 217882 & 423 & 432 & 314 & 615 & 488 & 635 & 6 & 1874 & 0 & 1268 & 1277 \\
\hline MHA_SEA chr16 6 & 218278 & 218461 & 2400 & 3767 & 2932 & 8284 & 5802 & 6790 & 24 & 12045 & 29 & 12269 & 11966 \\
\hline THA_SEA chr16 & 218449 & 218636 & 56 & 95 & 122 & 310 & 267 & 202 & 1 & 450 & 4 & 382 & 507 \\
\hline IHA_SEA chrr 6 & 218625 & 218838 & 79 & 161 & 189 & 461 & 454 & 321 & 1 & 575 & 0 & 566 & 630 \\
\hline IHA_SEA chr16 & 218706 & 218932 & 106 & 178 & 154 & 425 & 413 & 307 & 1 & 365 & 1 & 509 & 645 \\
\hline THA_SEA chr16 & 218921 & 219129 & 738 & 1307 & 774 & 2090 & 1664 & 1757 & 1 & 2906 & 6 & 2860 & 2983 \\
\hline HHA_SEA chr16 & 219111 & 219285 & 161 & 206 & 237 & 598 & 487 & 417 & 0 & 937 & 1 & 823 & 883 \\
\hline THA_SEA chrl 16 & 219274 & 219472 & 330 & 613 & 1017 & 2431 & 1875 & 1663 & 2 & 3105 & 11 & 3464 & 3236 \\
\hline THA_SEA chr16 & 219445 & 219598 & 173 & 267 & 283 & 613 & 518 & 520 & 1 & 1011 & 3 & 1001 & 1010 \\
\hline HA_SEA chr16 & 219524 & 219749 & 13 & 22 & 14 & 44 & 30 & 38 & 0 & 108 & 0 & 82 & 109 \\
\hline IHA_SEA chr16 & 219738 & 219900 & 551 & 701 & 783 & 1721 & 1412 & 1241 & 5 & 2387 & 3 & 2016 & 2636 \\
\hline THA_SEA chrl 16 & 219856 & 220064 & 137 & 188 & 384 & 746 & 625 & 636 & 1 & 1077 & 1 & 903 & 1388 \\
\hline IHA_SEA chrl 16 & 220061 & 220164 & 913 & 1085 & 1520 & 2123 & 1982 & 2093 & 6 & 4793 & 3 & 3244 & 3666 \\
\hline THA_SEA chr16 & 220152 & 220362 & 479 & 746 & 1006 & 2209 & 1885 & 1790 & 10 & 3049 & 1 & 2877 & 3091 \\
\hline HH_SEA chr16 6 & 220341 & 220490 & 458 & 603 & 811 & 1442 & 1241 & 1219 & 6 & 2391 & 7 & 1935 & 2106 \\
\hline THA_SEA chrr16 & 220838 & 220923 & 544 & 602 & 511 & 774 & 878 & 1067 & 5 & 2461 & 2 & 1914 & 1872 \\
\hline THA_SEA chrr16 & 20012 & 221126 & 539 & 908 & 1317 & 2751 & 2446 & 2418 & 6 & 4098 & 10 & 3604 & 4259 \\
\hline
\end{tabular}

Figure $2 b$

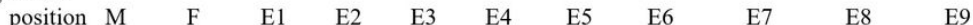

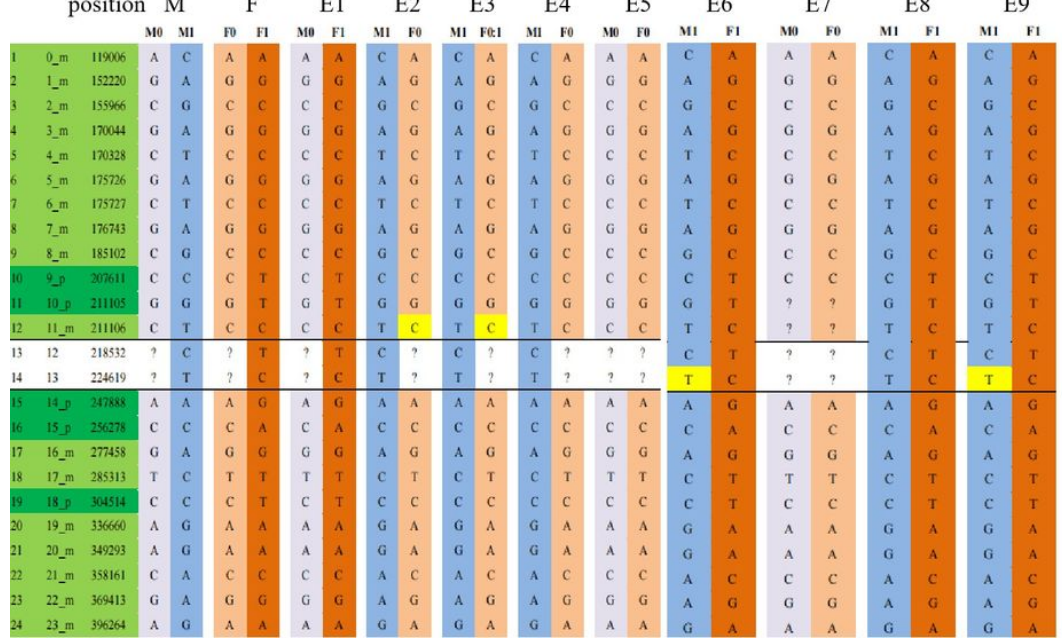

Figure 2

Case 13 presented as an example analysis of a- thalassemia (part of the SNP results). A. Sequencing depth of the SEA area. B. SNPs in the SEA area were used to construct the haplotype. The positions marked in dark green are paternal informative SNPs while those in light green are maternal informative SNPs. The yellow squares represent ADO sites. M0 and F0 represent an affected haplotype from mother and father, respectively. M1 and F1 indicate the normal inherited allele. A question mark represents the undetected site in the SEA area.

M: mother, F: father, E: embryo. 
Figure 3a

\begin{tabular}{l|l|l|l|} 
& \multicolumn{3}{|l|}{ chr11:5247992 CD41/42delAAAG } \\
No. & Depth & Genotype & Base_Depth \\
M & 1310 & C/C- & CAAAG(649)C (661) \\
F & 833 & C/C- & CAAAG (427)C (405) \\
E1 & 3074 & C/C- & CAAAG (1570)C (1503) \\
E2 & 573 & C/C- & CAAAG (245)C (328) \\
E3 & 3452 & C/C & C (3444) \\
\hline E4 & 2463 & C-/C- & CAAAG (12)C (2449) \\
E5 & 2965 & C/C- & CAAAG (1002)C (1962) \\
E6 & 4942 & C-/C- & CAAAG (25)C (4917) \\
\hline
\end{tabular}

Figure $3 b$

\begin{tabular}{|c|c|c|c|c|c|c|c|}
\hline M & $\mathrm{F}$ & E1 & E2 & E3 & E4 & E5 & E6 \\
\hline M0 M1 & F0 F1 & M0 F1 & M1 F0 & M1 F1 & M0 F0 & M0 F1 & M0 F0 \\
\hline
\end{tabular}

\begin{tabular}{|c|c|c|c|c|c|c|c|c|c|c|c|c|c|c|c|c|}
\hline 155_m 5951091 & A & G & A & A & A & A & G & A & G & A & A & A & A & A & A & A \\
\hline 156 _m 5959848 & A & G & A & A & A & A & G & A & G & $\mathrm{A}$ & A & A & A & A & A & A \\
\hline 157 -m 5959920 & c & G & C & c & c & c & G & C & G & c & c & c & c & c & c & C \\
\hline 158_m5967947 & c & $\mathrm{T}$ & c & c & c & $\mathrm{c}$ & $\mathrm{T}$ & c & $\mathrm{T}$ & $\mathrm{c}$ & c & c & c & c & c & c \\
\hline p 6014552 & G & G & G & A & G & $\mathrm{A}$ & G & G & G & $\mathrm{A}$ & G & G & G & A & G & G \\
\hline p 6014579 & G & G & G & A & G & A & G & G & G & A & G & G & G & A & G & G \\
\hline _p 6014611 & G & G & G & A & G & A & G & G & G & A & G & G & G & A & G & G \\
\hline 162 m 6035038 & G & A & G & G & G & G & A & G & A & G & G & G & G & G & G & G \\
\hline p 6049318 & A & A & c & A & A & A & A & c & A & A & A & c & A & A & A & c \\
\hline 6060247 & $\mathrm{~T}$ & $\mathrm{~T}$ & c & $\mathrm{T}$ & $\mathrm{T}$ & $\mathrm{T}$ & $\mathrm{T}$ & c & $\mathrm{T}$ & $\mathrm{T}$ & $\mathrm{T}$ & c & $\mathrm{T}$ & T & $\mathrm{T}$ & c \\
\hline _p6069067 & c & c & G & $\mathrm{c}$ & c & c & c & G & c & c & $\mathrm{c}$ & G & c & c & c & G \\
\hline$Z^{p 6083947}$ & c & c & $\mathrm{A}$ & c & C & c & C & A & C & c & c & A & c & c & c & A \\
\hline 67 p 6083952 & A & A & G & A & A & A & A & G & A & A & A & G & A & A & A & G \\
\hline 8 - 6084034 & G & G & $\mathrm{T}$ & G & G & G & G & $\mathrm{T}$ & G & G & G & $\mathrm{T}$ & $G$ & G & G & $\mathrm{T}$ \\
\hline 69_p 6239344 & G & G & G & A & G & A & G & G & G & A & G & G & G & A & G & G \\
\hline
\end{tabular}

Figure 3

Case 30, presented as an example analysis of $\beta$ - thalassemia. A. Sequencing depth of the CD 41-42 area. B. SNPs near the CD 41-42 area were used to construct the haplotype (part of the SNP results). The positions marked in dark green are paternal informative SNPs while the positions in light green are maternal informative SNPs. The yellow squares represent ADO sites. M0 and F0 represent an affected haplotype from the mother and father, respectively. $\mathrm{M} 1$ and $\mathrm{F} 1$ indicate the normal inherited allele.

M: mother, F: father, E: embryo.

\section{Supplementary Files}

This is a list of supplementary files associated with this preprint. Click to download.

- Supplementaltable.doc 